\title{
The Emergency Department Action in Smoking Cessation (EDASC) Trial: Impact on Cessation Outcomes
}

\author{
David A. Katz MD, MSc ${ }^{1,3,7}$, John E. Holman MS ${ }^{1,7}$, Andrew S. Nugent MD ${ }^{2}$, Laurence J. Baker DO ${ }^{8}$, \\ Skyler R. Johnson $\mathrm{MS}^{7}$, Stephen L. Hillis $\mathrm{PhD}^{4,5,7}$, David G. Tinkelman $\mathrm{MD}^{9}$, Marita G. Titler PhD, $\mathrm{RN}^{10}$, \\ Mark W. Vander Weg $\mathrm{PhD}^{1,6,7}$
}

${ }^{1}$ Department of Medicine, University of Iowa, Iowa City, IA; ${ }^{2}$ Department of Emergency Medicine, University of Iowa, Iowa City, IA; ${ }^{3}$ Department of Epidemiology, University of Iowa, Iowa City, IA $;{ }^{4}$ Department of Biostatistics, University of Iowa, Iowa City, IA; ${ }^{5}$ Department of Radiology, University of Iowa, Iowa City, IA; ${ }^{6}$ Department of Psychology, University of Iowa, Iowa City, IA ${ }^{7}$ Comprehensive Access \& Delivery Research and Evaluation (CADRE) Center, Iowa City VA Hospital, Iowa City, IA; ${ }^{8}$ Department of Emergency Medicine, Iowa Methodist Medical Center, Des Moines, IA; ${ }^{9}$ National Jewish Health, Denver, CO; ${ }^{10}$ University of Michigan School of Nursing, Ann Arbor, MI

Corresponding Author: David A. Katz, M.D., M.Sc., Center for Comprehensive Access \& Delivery Research and Evaluation (CADRE), VA Iowa City Health Care System (152), Building 42, Room 151, 601 Highway 6 West, Iowa City, IA 52246-2208, USA. Telephone: 319-338-0581, ext.7598; Fax: 319-887-4932; E-mail: david-katz@uiowa.edu

ClinicalTrials.gov registration number: NCT00756704 (date of submission 9/19/08). IRB approval: University of Iowa (6/25/08), Iowa Methodist Medical Center (11/13/08). This manuscript is the result of work supported in part with resources and the use of facilities at the Department of Veterans Affairs Medical Center, Iowa City, IA.

Received April 5, 2012; accepted September 4, 2012

\begin{abstract}
Introduction: The focus on acute care, time pressure, and lack of resources hamper the implementation of smoking cessation guidelines in the emergency department (ED). The purpose of this study was to determine whether an emergency nurseinitiated intervention based on the 5A's (Ask-Advise-Assess-Assist-Arrange) framework improves quit rates.

Methods: We conducted a pre-post implementation trial in 789 adult smokers who presented to two EDs in Iowa between August 13, 2008 and August 4, 2010. The intervention focused on improving delivery of the 5A's by ED nurses and physicians using academic detailing, charting/reminder tools, and group feedback. Performance of ED cessation counseling was measured using a 5A's composite score (ranging from 0 to 5). Smoking status was assessed by telephone interview at 3- and 6-month follow-up (with biochemical confirmation in those participants who reported abstinence at 6-month follow-up).

Results: Based on data from 650 smokers who completed the post-ED interview, there was a significant improvement in the mean 5A's composite score for emergency nurses during the intervention period at both hospitals combined (1.51 vs. 0.88 , difference $=0.63,95 \%$ confidence interval $[\mathrm{CI}][0.41,0.85])$. At 6-month follow-up, 7-day point prevalence abstinence (PPA) was 6.8 and $5.1 \%$ in intervention and preintervention periods, respectively (adjusted odds ratio $[O R]=1.7,95 \% C I[0.99,2.9]$ ).

Conclusions: It is feasible to improve the delivery of brief smoking cessation counseling by ED staff. The observed improvements in performance of cessation counseling, however, did not translate into statistically significant improvements in cessation rates. Further improvements in the effectiveness of ED cessation interventions are needed.
\end{abstract}

\section{INTRODUCTION}

There is growing recognition of the importance of smoking cessation in patients who present to the emergency department (ED). The prevalence of tobacco use in ED patients exceeds that of the general population (Lowenstein et al., 1998), and an estimated $4.9 \%$ of all ED visits and $10 \%$ of all hospital charges are attributable to tobacco (Bernstein \& Becker, 2002). Many smokers in the ED experience a heightened interest in quitting (McBride, Emmons, \& Lipkus, 2003), and are receptive to cessation counseling during or after the ED visit (Boudreaux, Baumann, Friedman, \& Ziedonis, 2005; Ersel et al., 2010; Klinkhammer, Patten, Sadosty, Stevens, \& Ebbert, 2005). Based on the U.S. Public Health Service (USPHS) clinical practice guideline (Fiore et al., 2008) and level I evidence in primary care settings, a public health task force convened by the American College of Emergency Physicians strongly recommended implementation of smoking cessation counseling in the ED setting (Bernstein et al., 2006). Most emergency physicians, however, do not consistently provide cessation counseling during ED visits (Bernstein et al., 2009). 
One approach to ensure more consistent smoking cessation counseling of ED patients is to redesign work processes to expand the clinical role of nurses (Stone et al., 2002) and to provide skills training in cessation counseling. Nursedelivered cessation counseling has been associated with higher quit rates as compared with usual care in a wide variety of non-ED practice settings (Mojica et al., 2004), and may also "prime" patients for physician counseling and prescription of pharmacotherapy (Duncan, Stein, \& Cummings, 1991). To address several known barriers to implementation of smoking cessation guidelines in the ED, we designed a pragmatic, ED nurse-initiated intervention based on the 5A's framework (Ask-Advise-Assess-Assist-Arrange) (Fiore et al., 2008; Whitlock, Orleans, Pender, \& Allan, 2002). The feasibility of training ED nursing staff to deliver brief cessation counseling has been previously demonstrated (Katz et al., 2012; Mahabee-Gittens, Gordon, Krugh, Henry, \& Leonard, 2008), but whether ED nurse-initiated counseling increases quit rates is unknown. Thus, the purpose of this analysis is to determine the effectiveness of a multifaceted intervention to improve onsite delivery of the 5A's by ED staff with regard to 3- and 6-month quit rates.

\section{MATERIALS AND METHODS}

\section{Study Design}

This study was designed as a pre-post guideline implementation trial in two EDs in Iowa: one University hospital that has a residency training program in Emergency Medicine (Hospital 1) and one large community teaching hospital that contracts with a group of private practice physicians to provide emergency medical services (Hospital 2). An advantage of this design is that each site serves as its own control (Glasgow, Magid, Beck, Ritzwoller, \& Estabrooks, 2005). During the preintervention period (which lasted 3-5 months at each site), ED staff performed their usual duties and the research team worked concurrently with ED leaders to develop practice tools for guideline implementation. At the outset of the intervention period (which also lasted 3-5 months at each site), study personnel trained ED nurses and physicians on how to implement the USPHS smoking cessation guideline. We monitored the data for time-related changes in cessation counseling (e.g., secular trends related to new hospital policies, maturation effects related to the evolution of clinical skills; Shadish et al., 2002), and regularly consulted with ED leaders to identify concurrent changes in practice that might affect our results. This project was approved by the institutional review board at each study hospital.

\section{Selection of Participants}

We enrolled a convenience sample of adult smokers, aged 18 years or older, who smoked at least 5 cigarettes per day and who presented to the ED on a walk-in basis or by private vehicle between August 13, 2008 and August 4, 2010 (see Figure 1 for exclusion criteria). Although very light smokers can also benefit from quitting, we excluded those who smoked less than 5 cigarettes per day. All eligible patients were invited to participate in the study regardless of their willingness to quit.

\section{Intervention}

Adapting elements of the Chronic Care Model (Glasgow, Orleans, \& Wagner, 2001; Revell \& Schroeder, 2005; Wagner, Austin, \& Von Korff, 1996), we envisioned the ED as the point of entry into a comprehensive smoking cessation program that extends beyond the ED visit (Figure 2). ED staff were trained to provide brief cessation counseling that was tailored to the patient's readiness to quit. As the aim of the present analysis is to evaluate cessation outcomes, we focus on those aspects of the intervention that relate to cessation counseling. More detail regarding other components of the study intervention are presented elsewhere (Katz et al., 2012).

\section{ED Staff Training}

Individualized face-to-face training was provided by the principal investigator (D.A.K.) to registered nurses in the ED (including those on the night shift), using an academic detailing approach (Goldstein et al., 2003; O'Brien et al., 2007), which has been shown to increase the volume of quitline (QL) referrals in primary care (Sheffer et al., 2012). The duration of training was approximately $20 \mathrm{~min}$, and included role-play exercises and hands-on instruction on use of charting and referral tools. Both ED nurses and physicians also received a pocket card showing the intervention algorithm (Supplementary Figure 1), and were instructed to view an online smoking cessation tutorial developed by the study investigators. Guidance for acute care nurses in applying the 5A's has been developed (Green \& Briggs, 2006). Consistent with principles of motivational interviewing (Lai, Cahill, Qin, \& Tang, 2010), ED staff were trained how to recognize and manage resistance to behavior change (Miller \& Rollnick, 1991; Rollnick, Mason, \& Butler, 2000). ED nurses and physicians were expected to deliver the 5A's to all smokers, including those who smoked less than 5 cigarettes per day (who were excluded from the trial).

\section{Modified Intake Form and Quick Orders}

We worked with each study site to adapt the 5A's intake form so that it could be readily integrated into the workflow of ED staff. To facilitate prescribing of recommended pharmacotherapy, computerized "quick orders" for these medications (with prefilled dose, duration, and patient instructions) were created at each site.

\section{Self-management Support}

For those patients who were willing to quit smoking within 30 days, the nurse was instructed to complete a QL referral, which was faxed to Quitline Iowa by the study research assistant (RA). Prior to the patient's quit date, one of three designated QL counselors called to confirm the patient's readiness to quit and discussed preparations for quitting (Fiore et al., 2000; Tsoh et al., 1997); this initial session typically lasted $30 \mathrm{~min}$. None of the Quitline counselors were members of the research team; all were regular QL employees. To build confidence in quitting, the counselor discussed problem-solving (e.g., dealing with urges to smoke and high-risk situations) and coping with nicotine withdrawal symptoms (e.g., use of pharmacotherapy). Up to eight follow-up calls, which focused on relapse prevention, were made by the same study counselor using a relapsesensitive schedule following the quit date (Kenford et al., 1994; Zhu et al., 1996). After the initial counseling session, a report summarizing the patient's smoking history and cessation plan was sent to the patient's primary care clinician (if any). 
The EDASC trial

\section{Pre-intervention}

8/13/08-1/14/09: Hospital 1 Enrollment 7/11/09-12/15/09: Hospital 2 Enrollment

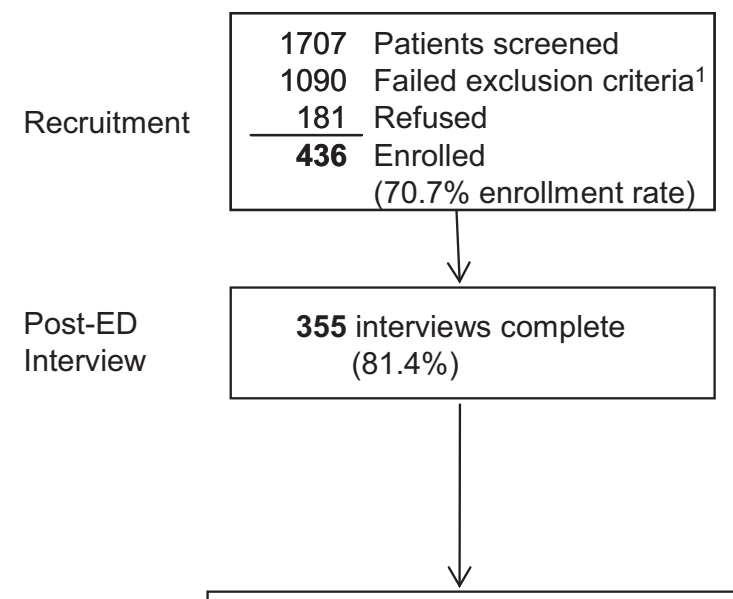

3-Month
follow-up

6-Month follow-up
436 Eligible for 3-month interview

280 3-month interviews complete (64.2\%)

0 interviews in progress

16 declined/withdrew

75 unable to locate ${ }^{2}$

65 unable to contact ${ }^{3}$

\section{Intervention}

2/17/09-5/1/09: Hospital 1 Enrollment 2/23/10-8/4/10: Hospital 2 Enrollment

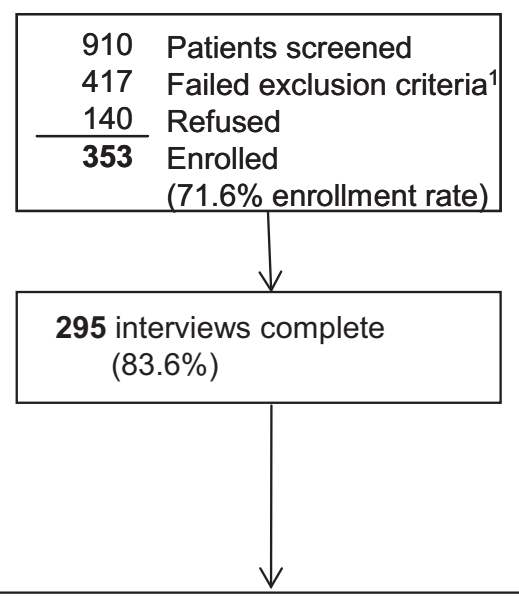

353 Eligible for 3-month interview

228 3-month interviews complete (64.9\%)

0 interviews in progress

18 declined/withdrew

50 unable to locate ${ }^{2}$

57 unable to contact ${ }^{3}$

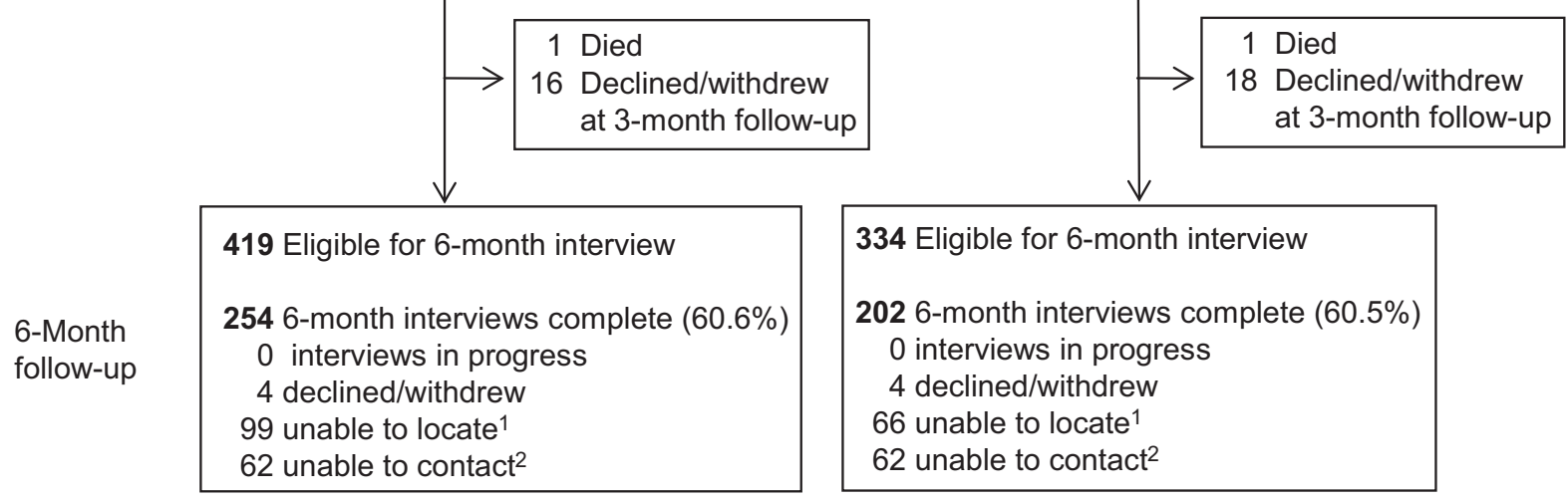

1. Exclusion criteria: acute medical decompensation (e.g., acute respiratory failure requiring intubation, cardiogenic or septic shock)(43), life-threatening trauma (1), altered mental status (22), dementia (0), language barrier (11), incarceration (0), transfer to another ED (4), departure from the ED prior to evaluation (46), inability to be contacted by telephone (32), uncontrolled psychiatric disorder or psychiatric emergency (0), sexual assault (0), prior enrollment in the study (15), intoxicated (7), other (26) <5 cigarettes/day (1300).

2. Unable to locate $=$ contact information on file no longer valid; unable to obtain updated contact information

3. Unable to contact $=$ interviewers were unable to complete interview despite having valid contact information

Figure 1. Study enrollment and follow-up.

Study patients who smoked at least 10 cigarettes per day, who had scheduled quit dates, and who had not yet obtained pharmacotherapy through other sources were eligible for a free 6-week course of nicotine replacement therapy (NRT, including patches, gum, or lozenges), which was mailed to the patient by Quitline Iowa (Miller et al., 2005). For those who reported having used NRT unsuccessfully in the past, were unable to tolerate it on account of side effects, or were ineligible for NRT, we encouraged emergency physicians to prescribe alternative pharmacotherapy (e.g., bupropion, varenicline), if appropriate. 


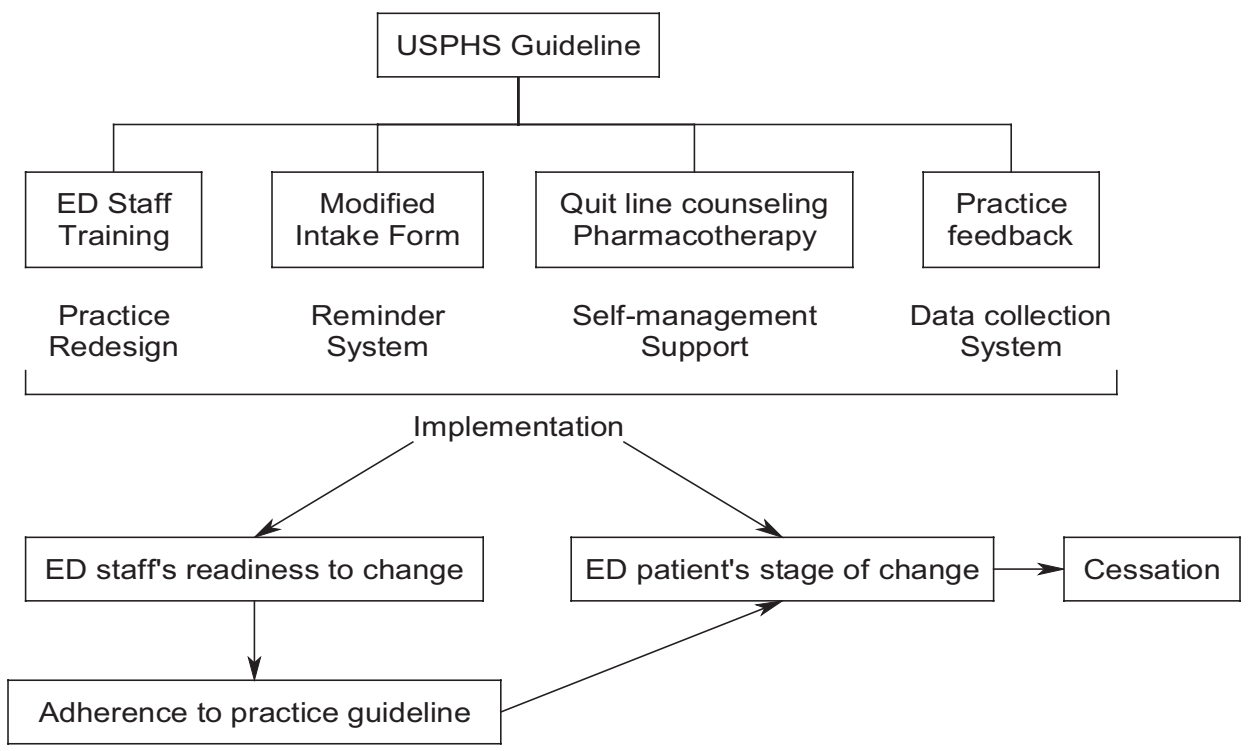

Figure 2. Conceptual model for the study intervention. ED = emergency department; USPHS = U.S. Public Health Service.

\section{Feedback to ED Staff}

Group feedback on performance of the 5A's was presented at the initial training session (based on data collected during preintervention period) and approximately every 2 months during the intervention period in small group meetings (nurses), business meetings (physicians), and via departmental newsletter and/or email. Practice feedback has been shown to be an important impetus for changing clinician behavior with regard to cessation counseling in primary care (Andrews, Tingen, Waller, \& Harper, 2001; Katz, Muehlenbruch, Brown, Fiore, \& Baker, 2004).

\section{Data Collection and Processing}

Every adult patient was screened for eligibility by medical record review and brief screening questionnaire by one of several study site RAs, who were stationed in the ED for a total of $40 \mathrm{~h}$ per week (between $7 \mathrm{AM}$ and $11 \mathrm{PM}$ ), including weekend days.

\section{Baseline Interview}

A baseline interview was administered to enrolled patients to assess sociodemographic factors, overall health status, any alcohol use, and smoking related variables (e.g., prior diagnosis of smoking-related illnesses [U.S. Department of Health and Human Services, 2010], patient's perceived relatedness of the ED encounter to smoking [Boudreaux et al., 2005], readiness to quit smoking [Contemplation Ladder; Biener \& Abrams, 1991], Fagerström Test for Nicotine Dependence [FTND; Heatherton, Kozlowski, Frecker, \& Fagerstrom, 1991]). Alcohol use was positive if the patient indicated that he or she had drunk any alcoholic beverages in the last 3 months. We assessed depressed mood using the PHQ-9 depression module (Kroenke, Spitzer, \& Williams, 2001).

\section{Postvisit Survey}

Shortly after leaving the ED (or transfer to an inpatient ward), the RAs telephoned study patients to determine whether or not the ED staff had performed the 5A's. Patient self-report of cessation counseling (within 2 weeks of primary care visits) has been shown to be highly accurate relative to audio taped interviews (Ward \& Sanson-Fisher, 1996), and is superior to medical record review (Conroy et al., 2005).

We calculated a 5A's composite score for each patient based on the sum of ask, assess, advise, assist, and arrange follow-up (ranging from 0 to 5, where each item was scored as 0 or 1), using a modified instrument originally developed to measure cessation counseling by inpatient nurses (Berndt et al., in press). Validity of the 5A's composite score is supported by the finding that patients were more likely to report abstinence at 6-months when more of the 5A's were delivered by either emergency nurses or physicians $(p=.003$, test for trend; Supplementary Table 1). In logistic regression models adjusting for readiness to quit, a 1-point increase in the 5A's composite score for emergency nurses and physicians was associated with a $33(95 \%$ confidence interval $[\mathrm{CI}][8,64])$ and $41(95 \% C I[14,76])$ percent increase in the odds of abstinence at 6-month follow-up, respectively.

Smoking Behavior at Follow-up

At 3- and 6-month follow-up, research staff who were not involved in delivering the study intervention contacted patients by telephone to ascertain current smoking status and any quit attempts $(>24 \mathrm{~h})$ during follow-up. All interviewers were blinded to assessment period, and administered study questionnaires using a script. A reminder letter was mailed to study patients approximately 2 weeks prior to each scheduled followup. Up to 15 attempts were made until successful contact was made.

Given the possibility that some ED patients may be inclined to overreport abstinence (e.g., those with smoking-related conditions), we mailed a collection kit for salivary cotinine determination to all participants who reported abstinence at 6-month follow-up (SRNT Subcommittee on Biochemical Verification, 2002). We asked these subjects about current use of NRT, as this could result in a false positive cotinine sample in a recent quitter (SRNT Subcommittee on Biochemical Verification, 2002). Cotinine assays were performed by J2 Laboratories (Tuscon, 


\section{The EDASC trial}

AZ) using liquid chromatography/tandem mass spectrometry (LC/MS/MS); a cutoff of $20 \mathrm{ng} / \mathrm{ml}$ was used to determine abstinence, as this threshold is associated with high sensitivity and specificity (>90\%; Etter, Vu Duc, \& Perneger, 2000).

\section{Outcome Measures}

We determined point prevalence abstinence (PPA) based on self-report of no smoking over 7 days prior to each interview at 3- and 6-month follow-up (Hughes et al., 2003; Velicer, Prochaska, Rossi, \& Snow, 1992). As PPA probably overestimates the proportion of smokers who will remain abstinent over time (Pierce \& Gilpin, 2003), we also report the repeated PPA (defined as no smoking over 7 days prior to both 3- and 6-month follow-up assessments; Hughes et al., 2003).

\section{Statistical Analysis}

Primary Data Analysis

We originally estimated that enrollment of 974 patients would provide approximately $80 \%$ power to detect a 6 percentagepoint absolute difference in 7-day PPA at 6-month follow-up, assuming that $10 \%$ of participants in the preintervention period would report abstinence, that $20 \%$ of enrollees would be lost to follow-up, and that the intraclass (i.e., within-nurse) correlation for self-reported abstinence was 0.015 (Katz et al., 2004), with a two-sided type I error of 0.05 . We did not attain this enrollment target for two reasons: (a) premature termination of enrollment at Hospital 1 on account of implementation of a new electronic medical record (with disablement of the computerized nurse reminder), and (b) a higher than expected refusal rate $(29 \%)$.

Preintervention and intervention groups were compared with respect to potential confounding variables using the twoindependent-sample $t$-test, Wilcoxon's rank-sum, or chi-square tests as appropriate. We also checked for any linear time trends in quit attempts during the preintervention period at each site, in order to detect any secular trends in ED patients' quitting behavior. Linear models of the 5A's composite score were estimated using generalized estimating equations with an independent working correlation matrix across the preintervention and intervention periods (Zeger \& Liang, 1986), adjusting for age, gender, race, education, presence of a smoking-related condition, patient concern that the ED symptoms might be smoking-related, cigarettes per day, readiness to quit, and study site. Regression assumptions of linearity and homoscedasticity were checked by inspection of residual plots and were sufficiently satisfied (Neter, Kutner, Nachtsheim, \& Wasserman, 1996).

We modeled 7-day PPA and repeated PPA outcomes, using logistic regression to adjust for study site and a set of prespecified covariates that have been associated with cessation in the literature: age, gender, cigarettes per day, tobacco dependence (FTND score), readiness to quit, and depression (Biener \& Abrams, 1991; Freund, D'Agostino, Belanger, Kannel, \& Stokes, 1992; Glassman et al., 1990; Hymowitz, Sexton, Ockene, \& Grandits, 1991; Piper, McCarthy, \& Baker, 2006; Thorndike et al., 2008). We used robust estimators of variance to account for clustering of outcomes at the ED nurse level (clustering was negligible for cessation outcomes; Huber, 1967). Model discrimination and calibration were acceptable for cessation outcomes (C-statistic ranged from 0.69 to 0.77 ); the Hosmer-Lemeshow goodness-of-fit test did not detect any significant lack of fit ( $p>.05$ for all models; Hosmer \& Lemeshow, 2000). All analyses were performed using an intent-to-treat approach in which participants were analyzed according to the treatment period during which they were originally enrolled (intervention vs. preintervention). In our primary analysis, participants who were unavailable for follow-up were considered to be smoking (penalized imputation).

\section{Secondary Analyses}

To evaluate differences in cessation rates between sites during the intervention period, we tested for interactions between period and site. We used multivariable logistic regression to determine whether the intervention increased the likelihood that patients made at least one serious quit attempt $(>24 \mathrm{~h}$ ) during follow-up. Lastly, we assessed whether intensity of cessation counseling (5A's composite score) was associated with 6-month PPA, by testing for a linear trend of the log odds (and adjusting for readiness to quit) across increasing composite score. We also estimated the impact of misreporting of abstinence by multiplying the 6-month PPA by the biochemical confirmation rate in both periods.

\section{Sensitivity Analysis}

The assumption that those lost to follow-up are still smoking may not necessarily provide a conservative estimate of intervention effect (Nelson, Partin, Fu, Joseph, \& An, 2009). Thus, we conducted a sensitivity analysis to evaluate missing data assumptions. First, we reanalyzed cessation outcomes in those patients who completed follow-up at 3 and 6 months (complete case analysis). Second, we used multiple imputation, which assumes that the data are "missing at random" (i.e., conditional on the value of other observed variables, whether an outcome is missing does not depend on the value of the outcome itself; Raghunathan, 2004). Of note, the proportion of study patients with missing follow-up data and the reasons for missingness (e.g., not reachable, declined interview) were virtually identical in both periods.

All statistical analyses were specified a priori in the study protocol, except for analysis of the 5A's composite scores (which was exploratory). We used STATA 10.0 (Stata Corp), SAS for Windows, version 9.2 (SAS Institute), and R programming language for all analyses. Multiple imputation was performed using the method of chained equations (Van Buuren, Boshuizen, \& Knook, 1999) as implemented by the STATA command ice (Royston, 2004). All tests were two-sided and a $p$-value of $<.05$ was defined as statistically significant; we did not adjust for multiple comparisons.

\section{RESULTS}

\section{Characteristics of Study Subjects}

Figure 2 summarizes the recruitment and follow-up of study patients. Those who were lost to 6-month follow-up $(N=333)$ were significantly younger (33 vs. 39 years, $p<.0001$ ), more likely to be nonwhite, $p=.03$ ), and less dependent on tobacco (mean FTND score 4.3 vs. $4.7, p=.04$ ) than those who completed 6-month follow-up (Supplementary Table 2). The mean ladder score was $6.0(S D=2.8)$, which roughly corresponds to the "contemplation" stage ("think I should quit but not quite ready"). As compared with preintervention patients, 
intervention patients were more likely to be female, more likely to have a smoking-related condition, and were more concerned that their acute symptoms might be related to smoking (Table 1). The Cochran-Armitage trend test showed no evidence of a secular trend in quit attempts during the preintervention period at either site (Agresti, 2002).

\section{Main Results}

Based on data from 650 smokers who completed the post-ED interview, there was a significant overall improvement in the mean 5A's composite score for emergency nurses (1.47 vs. 0.83 , adjusted difference $=0.68,95 \% C I[0.46,0.89])$ and for emergency physicians ( 1.15 vs. 0.76 , adjusted difference $=0.36,95 \%$ $C I[0.12,0.59])$ during the intervention period. Detailed results for the individual A's have been reported elsewhere (Katz et al., 2012).

Further analysis of assistive behaviors showed that 11 and $0 \%$ of intervention and preintervention period patients were referred to the QL, respectively (confirmed by checking internal QL records). In comparison, 42 and 38\% indicated that they were at least thinking about changing their smoking pattern (contemplation ladder scores $\geq 8$ ), respectively. Of the former group (i.e., motivated smokers during the intervention period), only $14 \%$ (20/148) were actually referred to the QL; this suggests that the pool of candidates for QL referral was considerably larger than those who were actually referred. In addition, 7\% (14/205) of less motivated patients (contemplation ladder scores $<8$ ) were referred to the QL. QL enrollees completed a mean of $4.6(S D=2.3$, range $=1-9)$ counseling sessions. Mean duration of total counseling time was $43.8 \mathrm{~min}$ $(S D=21.8)$; mean duration of the initial and aggregated follow-up counseling sessions was $16.5(S D=4.4)$ and 33.5 $(S D=15) \mathrm{min}$, respectively. Post-ED interview data showed that emergency physicians were more likely to discuss drug therapy for smoking cessation during the intervention period (as compared with preintervention): 18 and 5\% (adjusted $O R=4.8,95 \% C I[2.2,10.5], p<.0001)$, respectively. This change mirrored the increased frequency of nurses' discussion of drug therapy: 17 vs. $4 \%$, respectively (adjusted $O R=4.9$, $95 \%$ CI $[2.7,9.0], p<.0001)$.

Cessation outcomes at 3-and 6-month follow-up are shown in Table 2. The impact of the intervention was comparable at both sites (as evidenced by non-significant site by period interactions); thus, we report results for both hospitals combined. At 6-month follow-up, $6.8 \%$ of intervention patients reported 7-day PPA, as compared with $5.1 \%$ during the preintervention period (adjusted $O R=1.7,95 \% C I[0.99,2.9], p=.054$ ). Repeated PPA was 3.7 and $1.8 \%$, respectively (adjusted $O R=2.4,95 \% C I$ [6.1], $p=.08$ ). In addition, 6-month PPA was higher in patients with greater baseline readiness to quit (defined by a contemplation ladder score $\geq 8$ ), as compared with those with lower ladder scores ( 8.9 vs. $3.8 \%$, adjusted $\mathrm{OR}=2.1,95 \% C I=[1.1,3.8], p=.02)$, with no significant interaction between period and readiness to quit.

Although there was a trend toward higher quit rates during the intervention period, the proportion of intervention and preintervention patients who had made a quit attempt during 6-month follow-up was not significantly different (61.9 vs. $57.6 \%$, adjusted $O R=1.0,95 \% C I[0.72,1.5], p=0.80)$. Among those who were still smoking at 6-month follow-up, cigarette consumption had changed similarly (as compared with baseline) during both periods.

Table 1. Characteristics of ED Study Patients

\begin{tabular}{lcc}
\hline & Preintervention & Intervention \\
Variable & $(n=436)$ & $353)$ \\
\hline Age, mean $(S D)$ & $35.6(11.9)$ & $37.2(13.3)$ \\
Gender, \% male & 49 & $39^{*}$ \\
Race, $\%$ nonwhite & 12 & 11 \\
Highest grade, median $(I Q R)$ & $12(10-13)$ & $12(12-14)$ \\
Marital status, \% married or living with companion & 37 & 37 \\
Self-rated health, \% excellent-very good & 34 & 36 \\
Alcohol use in past 3 months, $\%$ & 62 & 58 \\
PHQ-9 depression score, median $(I Q R)^{\mathrm{a}}$ & $8(4-13)$ & $8(4-13)$ \\
Smoking-related variables & & $15(10-20)$ \\
Cigarettes per day, median $(I Q R)$ & $15(10-20)$ & $5(3-6)$ \\
Nicotine dependence (FTND), median $(I Q R)^{\mathrm{b}}$ & $5(3-6)$ & $38^{*}$ \\
Any smoking-related condition, $\%$ c & 31 & $26^{*}$ \\
Concern that ED symptoms might be related to smoking, $\%{ }^{\mathrm{d}}$ & 19 & $3(1-5)$ \\
Prior quit attempts $\left(>1\right.$ full day), median $(I Q R)^{\mathrm{e}}$ & $3(1-4)$ & $6.2(2.8)$ \\
Contemplation ladder $(0-10)$, mean $(S D)$ & $5.9(2.8)$ & 65 \\
Any other smoker in household, $\%$ & 60 & \\
\hline
\end{tabular}

Note. FTND = Fagerstrom Test of Nicotine Dependence; $I Q R=$ interquartile range; $S D=$ standard deviation.

${ }^{a}$ A PHQ-9 score of $>10$ has a sensitivity of $88 \%$ and a specificity of $88 \%$ in diagnosing major depression in primary care. Data were missing for 23 patients.

${ }^{b}$ Data were missing for 70 patients.

'Prior diagnosis of any of the following conditions: coronary heart disease, congestive heart failure, stroke or transient ischemic attack, chronic obstructive lung disease, peripheral vascular disease, tobacco-related cancer (e.g., lung, oral cavity).

dAt least "a little bit."

eResults shown for 346 and 258 preintervention and intervention period patients who reported any prior quit attempt, respectively. $* p<.05$. 


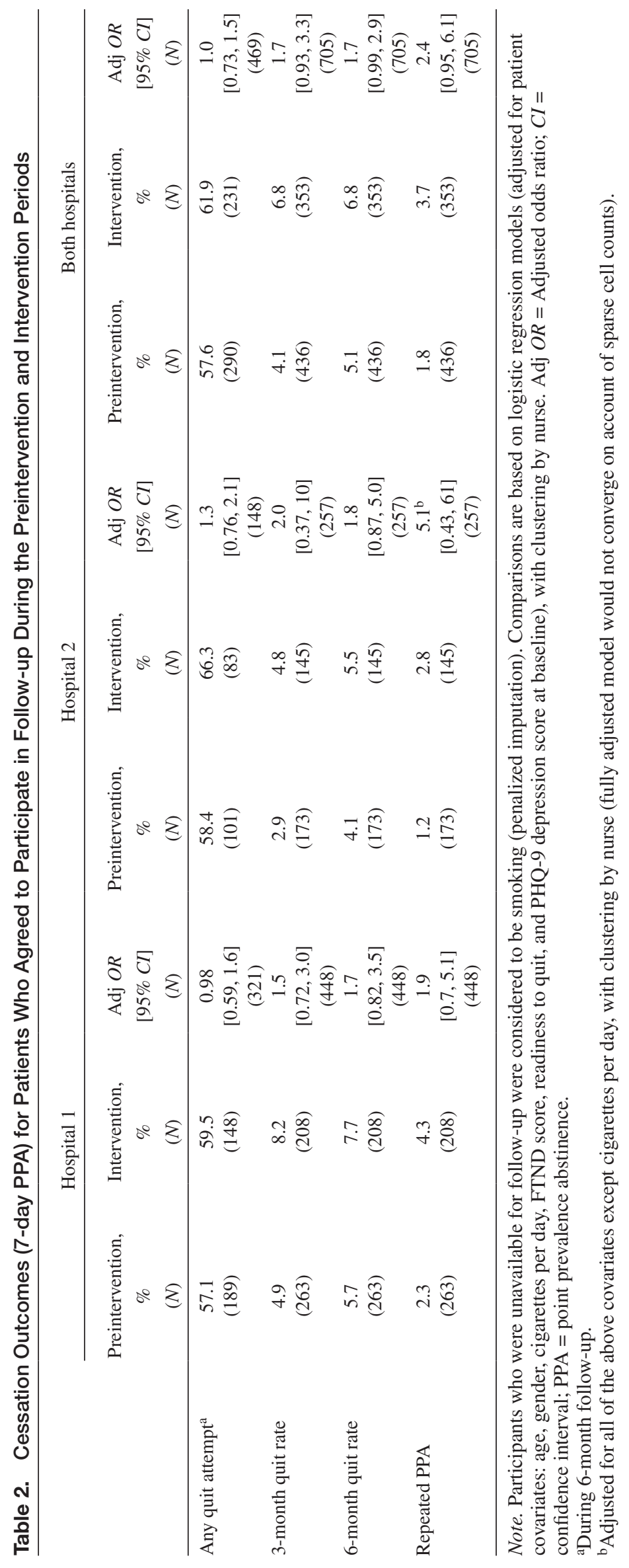


Among the 43 patients who reported abstinence at 6-month follow-up and agreed to provide a saliva sample (3 refused), the saliva sample return rates for intervention and preintervention patients were similar: 45\% (196/436) versus 43\% (152/353), respectively $(p=.87)$. Of the 15 patients with analyzable saliva samples, 67\% (6/9) of intervention patients and 67\% (4/6) of preintervention patients were confirmed to be abstinent from smoking (one preintervention patient was excluded as he reported using nicotine replacement therapy at the time of saliva collection).

\section{Sensitivity Analysis}

We explored the effect of missing cessation data on our findings. The results of complete case analysis were similar to those obtained using penalized imputation (base case); however, adjusted odds ratios for 6-month PPA and repeated PPA were distinctly lower (>10\%) when multiple imputation was used (Supplementary Table 3). We also assessed the impact of misreporting of abstinence; adjusting for the biochemical confirmation rate, the corrected 7-day PPA at 6-months was 4.6 and $3.4 \%$ in the intervention and preintervention periods, respectively.

\section{CONCLUSIONS}

In the $\mathrm{ED}$, the focus on acute care, time pressure, and the lack of an ongoing relationship with the patient may hinder the delivery of cessation counseling (Katz et al., 2010). By integrating cessation counseling into the nurse's workflow, our intervention was designed to enable ED staff to efficiently provide cessation counseling and pharmacotherapy based on the patient's readiness to quit. Study results demonstrate that patients enrolled during the intervention period were more likely to have received brief cessation counseling (based on the 5A's) from ED staff. In addition, ED staff were more likely to provide concrete assistance to those patients who were motivated to quit. The magnitude of improvement in the 5A's summary score was modest, however, and did not translate into statistically significant improvements in cessation rates. After adjustment for misreporting of abstinence, the difference in 6-month PPA between treatment groups was further attenuated.

The results of this study indicate that a substantial proportion of ED patients are considering smoking cessation (as indicated by mean contemplation ladder scores of 6 on a scale of $0-10$ ) and are receptive to low-intensity smoking cessation interventions in the ED. During the preintervention period, nearly $60 \%$ of study patients had made one or more quit attempts, and $5.7 \%$ reported having quit smoking at 6-month follow-up. The latter finding is comparable to that observed in the standard care arm of the EDITT trial (Boudreaux et al., 2008), which also enrolled unselected ED patients regardless of their willingness to quit.

Few published studies have addressed the delivery of smoking cessation interventions by ED nurses and physicians (Supplementary Table 4). Two randomized controlled trials in adults showed no benefit of brief physician advice coupled with referral for outpatient (face-to-face) tobacco use counseling (Antonacci \& Eyck, 2000; Richman et al., 2000); however, neither of these studies provided sufficient detail to judge whether the cessation intervention was implemented with high fidelity (Glasgow et al., 2005).
Several additional studies have investigated the feasibility and effectiveness of ED-based smoking cessation interventions using non-ED personnel to deliver the counseling intervention (Bernstein et al., 2011; Bock et al., 2008; Boudreaux et al., 2008; Neuner et al., 2009). Although interventions were delivered more consistently by trained research staff in these studies, cessation results were not much better than those relying upon ED personnel (Supplementary Table 4); moreover, these interventions were generally not sustainable as they relied exclusively on research staff. In some of these trials, cessation rates were higher in both study arms (as compared with those of the current trial), possibly secondary to the presence of a strong cue to action (e.g., chest pain; Bock et al., 2008), co-interventions (e.g., NRT; Bock et al., 2008; Bernstein et al., 2011), inclusion of more motivated patients (Bernstein et al., 2011), and selection bias (enrollment of patients who were more favorably disposed toward health promotion; Neuner et al., 2009).

There are several possible reasons why the intervention did not produce a larger effect on cessation rates. First, the proportion of patients who specifically received critical components of the intervention, such as advice to quit and QL referral, was low. Like their counterparts in primary care, some ED staff try to avoid confrontation with patients by giving advice only to those patients with smoking-related problems (Coleman, Cheater, \& Murphy, 2004). Second, we were unable to verify the quality of brief counseling as delivered by the ED staff; the data suggest that ED staff were no more effective in motivating patients to quit during the intervention, as the proportion of patients who made a quit attempt was similar in both periods. Third, several patients who were potential candidates for QL referral were not offered this service, and of those who were referred, many could not be reached by a telephone counselor or deferred counseling once they were contacted. These findings are similar to those obtained in feasibility trials of QL referral in ED patients (Mahabee-Gittens et al., 2008; Schiebel \& Ebbert, 2007). Fourth, most patients who were enrolled in QL counseling only participated in a single counseling session and did not receive booster calls. Prior research in inpatient smokers suggests that only interventions that include booster phone calls over a postdischarge period greater than 4 weeks lead to significantly greater cessation rates (Rigotti et al., 2012). Fifth, telephone follow-up revealed that several study patients who received a prescription for smoking cessation pharmacotherapy either did not fill their prescription or experienced administrative barriers in obtaining the medication. The ED smoking cessation algorithm included several interconnected steps during and after the ED visit - a lapse in any of these steps would tend to lessen the overall impact of the intervention.

Limitations of this trial deserve comment. First, we had reduced statistical power to detect the prespecified change in our primary outcome (6-month PPA) on account of low enrollment and higher than expected attrition; however, we still had $80 \%$ power to detect a 5.7 percentage-point difference (or an odds ratio of 2.25) in 7-day PPA at 6 months, based on the attained sample size and an observed preintervention quit rate of $5.1 \%$. Second, approximately $40 \%$ of study enrollees did not provide data for the 6-month follow-up survey, despite efforts to obtain complete contact information from subjects at enrollment (Thibodeau, Chan, Reilly, \& Reyes, 2000; Woolard et al., 2004) and to track down study patients with nonworking telephone numbers (Lovell \& Morcuende, 2006). 
High study attrition has been well-documented in ED populations (Boudreaux, Ary, St. John, \& Mandry, 2000; Schiebel \& Ebbert, 2007). The overall response rate was within the range reported for other practice-based studies $(>60 \%$; Hughes et al., 2003), however, and there was no evidence of differential loss to follow-up across periods. Third, only approximately half of self-reported quitters provided a saliva sample for biochemical confirmation of abstinence. Of those who did, abstinence was confirmed in only two-third of the subjects (which suggests that biochemical confirmation of abstinence is warranted in this population). Fourth, we did not collect data during low census hours (11 PM to 7 AM); however, demographics of the analysis sample were very similar to those of the two study EDs as a whole. Fifth, we excluded very light smokers on account of concern that these patients may have perceived the ED intervention to have less personal relevance. Considering that even low levels of smoking are associated with significant health risks, these patients should be included in future interventions in order to realize the full public health impact of smoking cessation efforts in the ED. Sixth, the modified 5A's summary score has not been validated in a separate patient population. Finally, the study was performed in two selected EDs in a single Midwestern state, and may not be generalizable to innercity or county EDs with a high proportion of uninsured patients or unselected EDs with less interest in smoking cessation.

Our findings challenge the prevailing guidance for ED staff to provide brief cessation counseling plus QL referral for the approximately 20 million smokers who visit the ED each year (Bernstein et al., 2007). In-depth interviews with ED staff suggest that current procedures for QL referral are cumbersome for ED staff (data not reported here); moreover, our data suggest that the impact of referral is attenuated by high rates of non-participation in the ED population. Although the study intervention did not attain statistical significance, it could nonetheless have a major public health impact (given its high reach, based on the estimated volume of smokers who present to U.S. EDs annually). Moreover, the difference between treatment groups in 6-month PPA $(1.7 \%)$ translates into a number-needed-to-treat (NNT) of 59; to put these results in perspective, the effectiveness of brief one-time physician advice during routine consultation, proactive telephone counseling, and self-help materials on long-term cessation ( $\geq 6$ months) has been estimated to be $2 \%(\mathrm{NNT}=50), 2.3 \%$ $(\mathrm{NNT}=43)$, and $1.5 \%(\mathrm{NNT}=67)$, respectively (Law \& Tang, 1995; Fiore et al., 2008).

Further work is needed to develop more effective ED cessation interventions that better integrate cessation counseling into ED work flow, that facilitate the real-time enrollment and retention of ED smokers in cessation counseling, and that reduce financial barriers to smoking cessation pharmacotherapy (without creating more hassles for ED clinicians). In addition, lessons can be learned from brief ED-based intervention trials that have been shown to reduce alcohol consumption in patients with hazardous and harmful drinking (D'Onofrio \& Degutis, 2002).

\section{SUPPLEMENTARY MATERIAL}

Supplementary Figure 1 and Tables $1-4$ can be found online at http://www.ntr.oxfordjournals.org

\section{FUNDING}

National Institute of Drug Abuse (R21 DA021607), Holden Comprehensive Cancer Center (Population Science Pilot Grant).

\section{DISCLAIMER}

The views expressed in this article are those of the authors and do not necessarily reflect the position or policy of the Department of Veterans Affairs or the U.S. government.

\section{DECLARATION OF INTERESTS}

None declared.

\section{ACKNOWLEDGMENTS}

The authors thank those who provided research assistance throughout the study: Sheryl Eastin, RN; Margaret Graham, MS; Rose Kim, MPH; Cassie Cunningham, MPH; Jennifer Carroll, RN; Yara Dahud, MS; Kadian Mijic, RN; and Maureen Myshock, MPH, MHA. George Bailey, MS, for assisting with database design, and Erin Greve for assisting with data entry. We are especially grateful for the assistance of the nurse facilitators, managers, and physician assistants who served as liaisons to the study team at each site: Shukrije Arifi, RN; Wendy Gerard, RN; Pat Bott, RN; Alfred Belinsky, RN; Elizabeth Finch, RN; Katie Iverson, PA; Rebecca Copeland, PA. We also thank Allison Anderson at the Iowa State University Center for Survey Statistics and Methodology for supervising collection of cessation data; Brittany Jackson and Allison Nuanes at National Jewish Health for coordination of Quitline counseling; and Mary Cherrico in the University of Iowa Prevention Intervention Center for assistance with project management, Lori Wright for administrative support, and Megan Watson for assistance with manuscript preparation.

\section{REFERENCES}

Agresti, A. (2002). Categorical data analysis. (2nd ed.). New York: Wiley. doi:10.1002/0471249688.

Andrews, J. O., Tingen, M. S., Waller, J. L., \& Harper, R. J. (2001). Provider feedback improves adherence with AHCPR smoking cessation guideline. Preventive Medicine, 33, 415421. doi:10.1006/pmed.2001.0907.

Antonacci, M. A., \& Eyck, R. T. (2000). Utilization and effectiveness of an emergency department initiated smoking cessation program [abstract]. Academic Emergency Medicine, 7, 1166.

Berndt, N. C., Bolman, C., de Vries, H., Segaar, D., van Boven, I., \& Lechner, L. (in press). Smoking cessation treatment practices recommendations for improved adoption on cardiology wards. Journal of Cardiovascular Nursing. doi:10.1097/JCN.0b013e318231f424.

Bernstein, S. L., Bernstein, E., Boudreaux, E. D., BabcockIrvin, C., Mello, M. J., \& Kapur, A. K. (2007). Public health considerations in knowledge translation in the emergency department. Academic Emergency Medicine, 14, 10361041. doi:10.1197/j/aem.2007.06.012. 
Bernstein, S. L., \& Becker, B. (2002). Preventive care in the emergency department: Diagnosis and management of smoking and smoking-related illness in the emergency department: A systematic review. Academic Emergency Medicine, 9, 720-729. doi:10.1197/aemj.9.7.720.

Bernstein, S. L., Bijur, P., Cooperman, N., Jearld, S., Arnsten, J. H., \& Moadel, A. (2011). A randomized trial of a multicomponent cessation strategy for emergency department smokers. Academic Emergency Medicine, 18, 575-583. doi:1.1111/ j.1553-2712-.2011.01097.x.

Bernstein, S. L., Boudreaux, E. D., Cabral, L., Cydulka, R. K., Schwegman, D., \& Larkin, G. L. et al. (2009). Efficacy of a brief intervention to improve emergency physicians' smoking cessation counseling skills, knowledge, and attitudes. Journal of Substance Abuse: Research and Treatment, 30, 158-181. doi:10.1080/08897070902802117.

Bernstein, S. L., Boudreaux, E. D., Cydulka, R. K., Rhodes, K. V., Lettman, N. A., \& Almeida, S.-L. (2006). Tobacco control interventions in the emergency department: A joint statement of emergency medicine organizations. Annals of Emergency Medicine, 48, e417-e425. doi:10.1016/j. annemergmed.2006.02.018.

Biener, L., \& Abrams, D. B. (1991). The contemplation ladder: Validation of a measure of readiness to consider smoking cessation. Health Psychology, 10, 360-365. doi:10.1037/0278-6133.10.5.360.

Bock, B. C., Becker, B. M., Niaura, R. S., Partridge, R., Fava, J. L., \& Trask, P. (2008). Smoking cessation among patients in an emergency chest pain observation unit: Outcomes of the chest pain smoking study (CPSS). Nicotine \& Tobacco Research, 10, 1523-1531. doi:10.1080114622200802326343.

Boudreaux, E. D., Ary, R. D., St. John, B., \& Mandry, C. V. (2000). Telephone contact of patients visiting a large, municipal emergency department: Can we rely on numbers given during routine registration? Journal of Emergency Medicine, 18, 409-415. doi:10.1016/S0736-4679(00)00155-4.

Boudreaux, E. D., Baumann, B. M., Friedman, K., \& Ziedonis, D. M. (2005). Smoking stage of change and interest in an emergency department-based intervention. Academic Emergency Medicine, 12, 211-218. doi:10.1197/j. aem.2004.09.022.

Boudreaux, E. D., Baumann, B. M., Perry, J., Marks, D., Francies, S., Camargo, C. A., Jr. (2008). Emergency department initiated treatments for tobacco (EDITT): A pilot study. Annals of Behavioral Medicine, 36, 314-325. doi:10.1007/ s12160-008-9066-3.

Coleman, T., Cheater, F., \& Murphy, E. (2004). Qualitative study investigating the process of giving anti-smoking advice in general practice. Patient Education \& Counseling, 52, 159-163. doi:10.1016/S0738-3991(03)00020-X.

Conroy, M. B., Majchrzak, N. E., Silverman, C. B., Chang, Y., Regan, S., \& Schneider, L. I. (2005). Measuring provider adherence to tobacco treatment guidelines: A comparison of electronic medical record review, patient survey, and provider survey. Nicotine \& Tobacco Research, 7, S35-S43. doi:10.1080/14622200500078089.

D'Onofrio, G., \& Degutis, L. C. (2002). Preventive care in the emergency department: Screening and brief intervention for alcohol problems in the emergency department: A systematic review. Academic Emergency Medicine, 9, 627-638. doi:10.1197/aemj.9.6.627.

Duncan, C., Stein, M. J., \& Cummings, S. R. (1991). Staff involvement and special follow-up time increase physicians' counseling about smoking cessation: A controlled trial. American Journal of Public Health, 81, 899-901. doi:10.2105/AJPH.81.7.899.

Ersel, M., Kitapcioglu, G., Solak, Z. A., Yuruktumen, A., Karahalli, E., \& Cevrim, O. (2010). Are emergency department visits really a teachable moment? Smoking cessation promotion in emergency department. European Journal of Emergency Medicine, 17, 73-79. doi:10.1097/ MEJ.0b013e32832e67d6.

Etter, J. F., Vu Duc, T., \& Perneger, T. V. (2000). Saliva cotinine levels in smokers and nonsmokers. American Journal of Epidemiology, 151, 251-258. doi:10.1046/j.1525-1497.2000.04119.x.

Fiore, M. C., Bailey, W. C., Cohen, S. J., Dorfman, S. F., Goldstein, M. G., \& Gritz, E. R. (2000). Treating tobacco use and dependence clinical practice guideline. Rockville, MD: U.S. Department of Health and Human Services, U.S. Public Health Service.

Fiore, M. C., Bailey, W. C., Cohen, S. J., Dorfman, S. F., Goldstein, M. G., \& Gritz, E. R. (2008). Treating tobacco use and dependence clinical practice guideline. Rockville, MD: U.S. Department of Health and Human Services, U.S. Public Health Service.

Freund, K. M., D'Agostino, R. B., Belanger, A. J., Kannel, W. B., \& Stokes, J., 3rd. (1992). Predictors of smoking cessation: The Framingham study. American Journal of Epidemiology, 135, 957-964. Retrieved from http://aje.oxfordjournals.org/.

Glasgow, R. E., Magid, D. J., Beck, A., Ritzwoller, D., \& Estabrooks, P. A. (2005). Practical clinical trials for translating research to practice: Design and measurement recommendations. Medical Care, 43, 551-557. doi:10.1097/01. mlr.0000163645.41407.09.

Glasgow, R. E., Orleans, C. T., \& Wagner, E. H. (2001). Does the chronic care model serve also as a template for improving prevention? Milbank Quarterly, 79, 579-612. doi:10.1111/1468-0009.00222.

Glassman, A. H., Helzer, J. E., Covey, L. S., Cottler, L. B., Stetner, F, \& Tipp, J. E. (1990). Smoking, smoking cessation, and major depression. Journal of the American Medical Association, 164, 1546-1549. doi:10.1001/ jama.264.12.1546.

Goldstein, M. G., Niaura, R., Willey, C., Kazura, A., Rakowski, W., \& DePue, J. (2003). An academic detailing intervention to disseminate physician-delivered smoking cessation counseling: Smoking cessation outcomes of the physicians counseling smokers project. Preventive Medicine, 36, 185-196. doi:10.1016/S0091-7435(02)00018-X.

Green, J. S., \& Briggs, L. (2006). Tobacco cessation in acute and critical care nursing practice: Challenges and approaches. Critical Care Nursing Clinics of North America, 18, 81-93. doi:10.1016/j.ccell.2005.11.005.

Heatherton, T. F., Kozlowski, L. T., Frecker, R. C., \& Fagerstrom, K. O. (1991). The Fagerstrom test for nicotine dependence: A revision of the Fagerstrom tolerance questionnaire. British Journal of Addiction, 86, 1119-1127. doi:10.1111/j.1360-0443.1991.tb01879.x.

Hosmer, D. W., \& Lemeshow, S. (2000). Applied logistic regression. (2nd ed.). New York: John Wiley \& Sons. doi:10.1002/ 0471722146.scard.

Huber, P. J. (1967). The behavior of maximum likelihood estimates under non-standard conditions. Paper presented at the Proceedings of the Fifth Berkeley Symposium on Mathematical Statistics and Probability, Berkeley, CA.

Hughes, J. R., Keely, J. P., Niaura, R. S., Ossip-Klein, D. J., Richmond, R. L., \& Swan, G. E. (2003). Measures of abstinence in clinical trials: Issues and recommendations. Nicotine \& Tobacco Research, 5, 13-25. doi:10.1093/ntr/5.1.13.

Hymowitz, N., Sexton, M., Ockene, J., \& Grandits, G. (1991). Baseline factors associated with smoking cessation and relapse. Preventive Medicine, 20, 590-601. doi:10.1016/0091-7435(91)90057-B.

Katz, D., Vander Weg, M., Holman, J., Nugent, A., Baker, L., \& Titler, M. (2010). Attitudes of 
emergency clinicians toward the delivery of smoking cessation counseling [abstract]. Paper presented at the Society for Medical Decision Making Annual Meeting, Toronto, ON, Canada. Retrieved from: http://smdm. confex.com/smdm/2010on/webprogram/Paper5864.html.

Katz, D. A., Muehlenbruch, D. R., Brown, R. L., Fiore, M. C., \& Baker, T. B. (2004). Effectiveness of implementing the agency for healthcare research and quality smoking cessation clinical practice guideline: A randomized controlled trial. Journal of the National Cancer Institute, 96, 594-603. doi:10.1093/jnci/djh103.

Katz, D. A., Vander Weg, M., Holman, J., Nugent, A., Baker, L., \& Johnson, S. (2012) Improving smoking cessation counseling in the emergency department: Results from the emergency department action in smoking cessation (EDASC) trial. Academic Emergency Medicine, 19, 409420. doi:10.1111/j.1553-2712.2012.01331.x.

Kenford, S. L., Fiore, M. C., Jorenby, D. E., Smith, S. S., Wetter, D., \& Baker, T. B. (1994). Predicting smoking cessation. Who will quit with and without the nicotine patch. Journal of the American Medical Association, 271, 589-594. doi:10.1001/jama.1994.03510320029025.

Klinkhammer, M. D., Patten, C. A., Sadosty, A. T., Stevens, S. R., \& Ebbert, J. O. (2005). Motivation for stopping tobacco use among emergency department patients. Academic Emergency Medicine, 12, 568-571. doi:10.1197/j. aem.2004.11.027.

Kroenke, K., Spitzer, R. L., \& Williams, J. B. (2001). The PHQ-9: Validity of a brief depression severity measure. Journal of General Internal Medicine, 16, 606-613. doi:10.1046/j.1525-1497.2001.016009606.x.

Lai, D. T., Cahill, K., Qin, Y., \& Tang, J. L. (2010). Motivational interviewing for smoking cessation. Cochrane Database of Systematic Reviews, 20, CD006936.

Law, M., \& Tang, J. L. (1995). An analysis of the effectiveness of interventions intended to help people stop smoking. Archives of Internal Medicine, 155, 1993-1941.

Lovell, M. E., \& Morcuende, J. A. (2006). Patient location strategies for pediatric long-term follow-up studies. Iowa Orthopaedic Journal, 26, 91-95. Retrieved from: http://www. ncbi.nlm.nih.gov/pmc/articles/PMC1888603/?tool=pubmed.

Lowenstein, S. R., Kosiol-McLain, J., Thompson, M., Bernstein, E., Greenberg, K., \& Gerson, L. W. (1998). Behavioral risk factors in emergency department patients: A multisite survey. Academic Emergency Medicine, 5, 781787. doi:10.1111/j.1553-2712.1998.tb02504.x.

Mahabee-Gittens, E. M., Gordon, J. S., Krugh, M. E., Henry, B., \& Leonard, A. C. (2008). A smoking cessation intervention plus proactive quitline referral in the pediatric emergency department: A pilot study. Nicotine \& Tobacco Research, 10, 1745-1751. doi:10.1080/1462220802443494.

McBride, C. M., Emmons, K. M., \& Lipkus, I. M. (2003). Understanding the potential of teachable moments: The case of smoking cessation. Health Education Research, 18, 156170. doi:10.1093/her/18.2.156.

Miller, N., Frieden, T. R., Liu, S. Y., Matte, T. D., Mostashari, F., \& Deitcher, D. R. (2005). Effectiveness of a large-scale distribution programme of free nicotine patches: A prospective evaluation. Lancet, 365, 1849-1854. doi:10.1016/ S0140-6736(05)66615-9.

Miller, W. R., \& Rollnick, S. (1991). Motivational interviewing. New York: Guilford Press.

Mojica, W. A., Suttorp, M. J., Sherman, S. E., Morton, S. C., Roth, E. A., \& Maglione, M. A. (2004). Smoking-cessation interventions by type of provider: A meta-analysis. American Journal of Preventive Medicine, 26, 391-401. doi:10.1016/j. amepre.2004.02.014.
Nelson, D. B., Partin, M. R., Fu, S. S., Joseph, A. M., \& An, L. C. (2009). Why assigning ongoing tobacco use is not necessarily a conservative approach to handling missing tobacco cessation outcomes. Nicotine \& Tobacco Research, 11, 7783. doi:10.1093/ntr/ntn013.

Neter, J., Kutner, M., Nachtsheim, C., \& Wasserman, W. (1996). Applied linear statistical models. (4th ed.). Homewood, IL: Irwin.

Neuner, B., Weiss-Gerlach, E., Miller, P., Martus, P., Hesse, D., \& Spies, C. (2009). Emergency department-initiated tobacco control: A randomised controlled trial in an intercity university hospital. Tobacco Control, 18, 283-293. doi:10.1136/ tc.2008.028753.

O'Brien, M. A., Oxman, A. D., Davis, D. A., Haynes, R. B., Freemantle, N., \& Harvey, E. L. (2007). Educational outreach visits: Effects on professional practice and health care outcomes. Cochrane Database of Systematic Reviews, 4, CD000409. doi:10.1002/14651858.CD000409.pub2.

Pierce, J. P., \& Gilpin, E. A. (2003). A minimum 6-month prolonged abstinence should be required for evaluating smoking cessation trials. Nicotine \& Tobacco Research, 5, 151-153. doi:10.1080/14622200307231.

Piper, M. E., McCarthy, D. E., \& Baker, T. (2006). Assessing tobacco dependence: A guide to measure evaluation and selection. Nicotine \& Tobacco Research, 8, 339-351. doi:10.1080/14622200600672765.

Raghunathan, T. E. (2004). What do we do with missing data? Some options for analysis of incomplete data. Annual Review of Public Health, 25, 99-117. doi:10.1146/annurev. publhealth.25.102802.124410.

Revell, C. C., \& Schroeder, S. A. (2005). Simplicity matters: Using system-level changes to encourage clinician intervention in helping tobacco users quit. Nicotine \& Tobacco Research, 7, S67-S69. doi:10.1080/14622200500078162.

Richman, P. B., Dinowitz, S., Nashed, A. H., Eskin, B., Sylvan, E., Allegra, C. (2000). The emergency department as a potential site for smoking cessation intervention: A randomized, controlled trial. Academic Emergency Medicine, 7, 348-353. doi:10.1111/j.1553-2712.2000.tb02235.x.

Rigotti, N. A., Clair, C., Manafo, M. R., \& Stead, L. F. (2012). Interventions for smoking cessation in hospitalised patients. Cochrane Database Systematic Reviews, 5, CD001837. doi:10.1002/14651858.CD001837.pub3.

Rollnick, S., Mason, P., \& Butler, C. (2000). Health behavior change: A guide for practitioners. New York: Churchill Livingstone.

Royston, P. (2004). Multiple imputation of missing values. Stata Journal, 4, 227-241.

Schiebel, N. E. E., \& Ebbert, J. O. (2007). Quitline referral vs. Self-help manual for tobacco use cessation in the emergency department: A feasibility study. BMC Emergency Medicine, 7(15). doi:10.1186/1471-227X-7-15.

Shadish, W. R., Cook, T. D., \& Campbell, D. T. (2002). Experimental and quasi-experimental designs for generalized causal inference. Boston: Houghton Mifflin.

Sheffer, M. A., Baker, T. B., Fraser, D. L., Adsit, R. T., McAfee, T. A., \& Fiore, M. C. (2012). Fax referrals, academic detailing, and tobacco quitline use: A randomized trial. American Journal of Preventive Medicine, 42, 21-28. doi:10.1016/j. amepre.2011.08.028.

SRNT Subcommittee on Biochemical Verification. (2002). Biochemical verification of tobacco use and cessation. Nicotine \& Tobacco Research, 4, 149-159. doi:10.1080/14622200210123581.

Stone, E. G., Morton, S. C., Hulscher, M. E., Maglione, M. A., Roth, E. A., \& Grimshaw, J. M. (2002). Interventions that increase use of adult immunization and cancer screening services: A meta-analysis. Annals of Internal Medicine, 136, 
641-651. Retrieved from: http://www.annals.org/content/ 136/9/641.full.

Thibodeau, L. G., Chan, L, Reilly, K. M., \& Reyes, V. M. (2000). Improving telephone contract rates of patients discharged from the emergency department. Annals of Emergency Medicine, 35, 564-567. doi:10.1016/ S0196-0644(00)70028-X

Thorndike, A. N., Regan, S., McKool, K., Pasternak, R. C., Swartz, S., \& Torres-Finnerty, N. (2008). Depressive symptoms and smoking cessation after hospitalization for cardiovascular disease. Archives of Internal Medicine, 168, 186-191. doi:10.1001/archinternmed.2007.60.

Tsoh, J. Y., McClure, J. B., Skaar, K. L., Wetter, D. W., Cinciripini, P. M., \& Prokhorov, A. V. (1997). Smoking cessation. 2: Components of effective intervention. Behavioral Medicine, 23, 15-27. doi:10.1080/08964289709596363.

U.S. Department of Health and Human Services. (2010). How tobacco smoke causes disease: The biology and behavioral basis for smoking-attributable disease: A report of the surgeon general. Atlanta, GA: Author.

Van Buuren, S., Boshuizen, H. C., \& Knook, D. L. (1999). Multiple imputation of missing blood pressure covariates in survival analysis. Statistics in Medicine, 18, 681-694. doi:10.1002/(SICI)1097-0258(19990330)18:6<681::AIDSIM71> 3.3.CO;2-I.

Velicer, W. F., Prochaska, J. O., Rossi, J. S., \& Snow, M. G. (1992). Assessing outcome in smoking cessation studies.
Psychological Bulletin, 111, 23-41. doi:10.1037//0033-2909. 111.1.23.

Wagner, E. H., Austin, B. T., \& Von Korff, M. (1996). Organizing care for patients with chronic illness. Milbank Quarterly, 74, 511-544. doi:10.2307/3350391.

Ward, J., \& Sanson-Fisher, R. (1996). Accuracy of patient recall of opportunistic smoking cessation advice in general practice. Tobacco Control, 5, 110-113. doi:10.1136/ tc.5.2.110.

Whitlock, E. P., Orleans, C. T., Pender, N., \& Allan, J. (2002). Evaluating primary care behavioral counseling interventions: An evidence-based approach. American Journal of Preventive Medicine, 22, 267-284. doi:10.1016/ S0749-3797(02)00415-4.

Woolard, R. H., Carty, K., Wirtz, P., Longabaugh, R., Nirenberg, T. D., \& Minugh, A. (2004). Research fundamentals: Follow-up of subjects in clinical trials: Addressing subject attrition. Academic Emergency Medicine, 11, 859-866. doi:10.1197/j.aem.2003.09.021.

Zeger, S. L., \& Liang, K. Y. (1986). Longitudinal data analysis for discrete and continuous outcomes. Biometrics, 42, 121130. doi: $10.2307 / 2531248$.

Zhu, S., Stretch, V., Balabanis, M., Rosbrook, B., Sadler, G., \& Pierce, J. (1996). Telephone counseling for smoking cessation: Effects of single-session and multiple-session interventions. Journal of Consuting \& Clinical Psychology, 64, 202-211. doi:10.1037//0022-006X.64.1.202. 